\title{
Effects of sardine oil and milk lipids on oxidative stress and inflammation markers in diet-induced hypercholesterolaemic rats
}

\author{
F. Dehiba, A. Benyahia-Mostefaoui, H. M. Khelladi, A. Boualga, M. Y. Lamri-Senhadji \\ and M. Bouchenak \\ Laboratoire de Nutrition Clinique et Métabolique, Faculté des Sciences, Université dOran Es Sénia, Oran 31000, Algeria
}

Free radicals in biological systems have been implicated in inflammation, atherosclerosis, and numerous other diseases and disorders. It is not clear that the source of lipid in the diet can modulate the inflammatory response to stress and disease. The aim of the study was to verify the effects of dietary lipids on dyslipidaemia and markers of oxidative stress and inflammation, in diet-induced hypercholesterolaemic rats.

Male Wistar rats $(n=18)$ weighing $200 \pm 10 \mathrm{~g}$ were fed diets (containing $20 \%$ casein enriched with $1.5 \%$ cholesterol) varying in lipid $(5 \%)$ sources and consisted of either casein-sardine oil (CASs), casein mixture of vegetable oils (olive $3.9 \%+$ nut $1 \%+$ sunflower $0.1 \%$, $n-6 / n-3=7$ (CASv), casein milk lipids (CASm) for 28 days. Serum total cholesterol and triacyglycerols were evaluated. Serum lipid peroxidation was measured by thiobarbituric acid reactive substances (TBARS) analysis ${ }^{(1)}$. Oxidized proteins were estimated by carbonyls concentrations ${ }^{(2)}$. Serum nitric oxide (NO) determination was performed using the Griess reagent (sulphanilamide and n-naphtyl-ethylenediamine $)^{(3)}$. Albumin and uric acid were determined by colorimetric methods. C-reactive protein (CRP) was assessed by Omega Diagnostics Kit.

Serum total cholesterol was 3.3- and 2.8-fold lower in CASm and CASv than in CASs group. TG values were decreased in CASs $v$. CASv $(-7 \%)$ and CASm $(-34 \%)$. There was no significant difference in serum TBARS values in CASs $v$. CASv and CASm groups. Increase in NO is noted in CASs compared to CASm and CASv (2.5-fold). There was no significant difference in NO values between CASm and CASv. Uric acid and albumin concentrations were elevated in CASs compared to CASm (1.6- and 1.4-fold) and CASv (2- and 1.6-fold), respectively. However, CRP, concentrations were higher in CASs $(P<0.05)$.

In conclusion, sardine oil involves higher level of total cholesterol than milk lipids diet. In contrast, an hypotriglyceridemic effect of sardine oil is observed in comparison with vegetable oils mixture and milk lipids. The sardine oil as well as the milk lipids protect the serum against the cytotoxic action and oxidative stress of cholesterol-enriched diet without attenuation of the inflammation.

1. Quintanilha AT, Packer L, Davies JM et al. (1982) Ann NY Acad Sci 393, 32-47.

2. Levine RL, Garland D, Oliver CN et al. (1990) Methods Enzymol 186, 464-478.

3. Cortas NK \& Wakid NW (1990) Clin Chem 36, 1440-1443. 